\title{
BMJ
}

\section{Isoniazid resistance and death in patients with tuberculous meningitis: retrospective cohort study}

\author{
Christopher Vinnard, fellow, ${ }^{1,2}$ Carla A Winston, senior epidemiologist, ${ }^{3}$ E Paul Wileyto, assistant professor, 2,4 \\ Rob Roy MacGregor, professor emeritus, ${ }^{1}$ Gregory P Bisson, assistant professor ${ }^{1,2}$
}

${ }^{1}$ Department of Medicine, Division of Infectious Diseases, University of Pennsylvania School of Medicine, 502 Johnson Pavilion, 3610 Hamilton Walk, Philadelphia, PA 19104, USA

${ }^{2}$ Department of Epidemiology and Biostatistics, Center for Clinical Epidemiology and Biostatistics,

University of Pennsylvania Schoo of Medicine

${ }^{3}$ Division of Tuberculosis

Elimination, Surveillance,

Epidemiology, and Outbreak

Investigations Branch, Centers for

Disease Control, Atlanta, GA

30333, USA

${ }^{4}$ Department of Psychiatry,

University of Pennsylvania School of Medicine

Correspondence to: C Vinnard christopher.vinnard@uphs.upenn. edu

Cite this as: $B M J$ 2010;341:C4451 doi:10.1136/bmi.c4451

\section{ABSTRACT}

Objective To determine whether initial isoniazid resistance is associated with death during the treatment of tuberculous meningitis.

Design Retrospective cohort study.

Setting National Tuberculosis Surveillance System at the Centers for Disease Control in the United States.

Participants Patients with a clinical diagnosis of tuberculous meningitis, reported to the National Tuberculosis Surveillance System between 1 January 1993 and 31 December 2005.

Main outcome measure All cause mortality during antituberculous treatment.

Results Between 1993 and 2005, 1896 patients had a clinical diagnosis of tuberculous meningitis and positive cultures from any site. In 123 (6\%) of these patients, isoniazid resistance was present on initial susceptibility testing. The unadjusted association between initial isoniazid resistance and subsequent death among these 1896 patients did not reach statistical significance (odds ratio $1.38,95 \%$ confidence interval 0.94 to 2.02 ).

However, among 1614 patients with positive cerebrospinal fluid cultures, a significant unadjusted association was found between initial isoniazid resistance and subsequent death (odds ratio 1.61, 1.08 to 2.40). This association increased after adjustment for age, race, sex, and HIV status (odds ratio 2.07, 1.30 to 3.29).

Conclusions Isoniazid resistance on initial susceptibility testing was associated with subsequent death among cases of tuberculous meningitis with positive cerebrospinal fluid cultures. Randomised controlled trials are needed to evaluate the optimal empirical regimen for treating patients with tuberculous meningitis who are at high risk for both initial isoniazid resistance and poor clinical outcomes.

\section{INTRODUCTION}

Tuberculous meningitis is the most devastating manifestation of tuberculosis. As a consequence of the merging of the HIV and tuberculosis epidemics, tuberculosis has become the most common cause of bacterial meningitis in some areas. ${ }^{12}$ Starting effective anti-tuberculous treatment early is essential for a successful outcome. ${ }^{3}$ Among the first line drugs, isoniazid is the only bactericidal agent that easily crosses the blood-brain barrier, achieving concentrations in cerebrospinal fluid similar to those in serum. ${ }^{45}$ Rifampicin, ethambutol, and streptomycin all penetrate cerebrospinal fluid poorly, even in the setting of meningeal inflammation. ${ }^{6-8}$ Pyrazinamide freely crosses the blood-brain barrier, but its role in tuberculous meningitis is uncertain given its bacteriostatic activity against older, resting bacilli. ${ }^{910}$ Isoniazid also has the strongest early bactericidal activity of all first line agents, defined as the reduction of bacillary load during the first two days of anti-tuberculous treatment. ${ }^{1112}$

Guidelines for the management of drug resistant tuberculosis are based on analysis of evidence from trials of pulmonary tuberculosis done by the British Medical Research Council, ${ }^{13}$ in which resistance to isoniazid was not associated with adverse outcomes when combination treatment with ethambutol, rifampicin, and pyrazinamide was used. This experience has informed recommendations for treatment of other forms of tuberculosis, including tuberculous meningitis. ${ }^{14}$ However, given important differences between tuberculous meningitis and pulmonary tuberculosis with respect to the anatomical locations of organisms, mechanisms of host injury, and rapidity of disease progression, the role of anti-tuberculous drug resistance in tuberculous meningitis deserves an independent examination. ${ }^{15}$

In other types of bacterial meningitis, bactericidal activity and penetration of cerebrospinal fluid are antimicrobial properties relevant for clinical cure. ${ }^{16}$ Therefore, isoniazid resistance in tuberculous meningitis may affect treatment outcomes to an extent that has not been seen for pulmonary tuberculosis. During the interval of time needed to obtain results from initial anti-tuberculous drug susceptibility testing (typically six to eight weeks), irreversible progression of disease may have occurred. Whereas previous studies of the association between initial isoniazid resistance and outcome of treatment have been limited by small sample sizes, ${ }^{17}$ the tuberculosis surveillance dataset maintained by the Centers for Disease Control in the United States offers a unique opportunity to study this relation. We tested the hypothesis that initial isoniazid resistance would be associated with adverse outcomes in 
patients being treated for an initial episode of tuberculous meningitis.

\section{METHODS}

\section{Setting}

We analysed data from the National Tuberculosis Surveillance System at the Centers for Disease Control, which has collected aggregate data on cases of tuberculosis since 1953 and patient specific data (including information on drug susceptibility) since 1993. State health departments report cases of tuberculosis by using the report of verified case of tuberculosis standard form, an anonymous reporting mechanism that consists of an initial report and two follow-up reports. ${ }^{18}$ A case must satisfy a standardised tuberculosis case definition to be included in the national count. A multi-state study that used multiple secondary sources to ascertain unreported cases found the completeness of the tuberculosis reporting system to be $99.5 \% .^{19}$

Clinical and demographic data are collected in the initial report as described extensively elsewhere. ${ }^{18}$ For each case with a positive culture or smear, the anatomical site is included in the initial report. The initial drug regimen is reported, along with the date that treatment started. For all states except California, HIV status is included in the report. For California, HIV status is obtained by linking with the California AIDS registry, which does not include HIV positive people without AIDS. No matching with the California AIDS registry has occurred since 2004. Results for patients with negative HIV tests are also unavailable for cases in California.

Approximately $80 \%$ of cases of tuberculosis reported to the Centers for Disease Control are culture positive, and approximately $97 \%$ of culture positive cases have accompanying drug susceptibility data, which are included in the first follow-up report. The second follow-up report includes the date treatment stopped and reasons for stopping treatment, which are "completed therapy," "moved," "lost," "uncooperative or refused," "not TB," "died," "other," and "unknown."

\section{Participants}

Our analysis examined data on all cases of tuberculosis reported between 1 January 1993 and 31 December 2005 , which allowed for at least two years of followup. We selected patients for inclusion if a meningeal site of involvement was reported and if they were alive at diagnosis and started anti-tuberculous treatment. We excluded patients with a previous diagnosis of tuberculosis, as we reasoned that these patients may be treated differently by clinicians on the basis of known or suspected drug resistance from previous treatment histories. To test the hypothesis that initial isoniazid resistance is associated with subsequent death, we limited the analysis to patients with a diagnosis of tuberculous meningitis, a positive culture for Mycobacterium tuberculosis from any site, and initial isoniazid susceptibility data. We excluded patients with initial resistance to both isoniazid and rifampicin (multi-drug resistance).

\section{Statistical methods}

We determined the unadjusted association between initial isoniazid resistance and subsequent death by using the $\chi^{2}$ test. We calculated an odds ratio and a $95 \%$ confidence interval to evaluate the strength of the association and the precision of the effect. We then stratified the association of initial isoniazid resistance and subsequent death according to whether the patient was infected with HIV and whether the patient had a positive cerebrospinal fluid culture.

We screened other demographic and clinical factors for inclusion in a multivariate logistic regression model on the basis of unadjusted associations with death $(\mathrm{P}<0.25)$. We treated age categorically because it was not normally distributed. Once we had identified variables for further evaluation in the multivariate model, we used multiple imputation for variables with missing observations. We determined clinical and demographic variables to have missing observations if the variable was listed as "not done" or "unknown" or had a missing response. Observations with missing values were not a random sample of the entire dataset, as is the case with "missing completely at random" data. Rather, whether certain clinical variables (such as HIV status) were missing depended on other variables in the dataset (such as age and race), creating a "missing at random" pattern. With such data, removing participants with missing observations from the analysis will lead to a biased sampling of the study population, and for this reason we chose multiple imputation as the approach to handle missing data. The final estimates in the multivariate model came from five imputed datasets. ${ }^{20}$

Variables remained in the model if they changed the odds ratio for isoniazid resistance and subsequent death by more than $15 \%$. For clinical reasons, sex and HIV coinfection remained in the final model, regardless of their effect on the association of interest. We evaluated whether a statistically significant interaction was present between HIV co-infection and initial isoniazid resistance, as well as between positive cerebrospinal fluid cultures and initial isoniazid resistance. We did a secondary analysis limited to adults initially treated with isoniazid, rifampicin (or rifabutin), pyrazinamide, and ethambutol. Finally, we did sensitivity analyses examining different approaches to handling missing HIV status, as well as different assumptions about the patients who were lost to follow-up. We used STATA v10.0 for all statistical calculations.

\section{RESULTS}

\section{Unadjusted analyses}

A total of 3114 cases of tuberculous meningitis were reported to the Centers for Disease Control between 1 January 1993 and 31 December 2005, and 3023 $(97 \%)$ occurred in people without a previous diagnosis of tuberculosis. After exclusion of 967 patients lacking positive cultures from any site, 131 patients without 
isoniazid susceptibility results, and 29 patients with multi-drug resistant disease, 1896 patients remained for analysis (figure).

Of these 1896 patients, 1614 (85\%) had a positive culture from cerebrospinal fluid and $282(15 \%)$ had positive cultures only from other sites. Five hundred and forty-one (29\%) patients died during treatment. Of the remaining 1355 patients, 1177 (87\%) completed treatment and $178(13 \%)$ were lost to follow-up. Among patients who completed treatment, the median duration of treatment was 366 days for isoniazid susceptible disease and 438 days for isoniazid resistant disease. For patients who died, the median duration of treatment was 37 days for isoniazid susceptible disease and 52 days for isoniazid resistant disease. For patients who were lost to follow-up, the median duration of treatment was 146 days for isoniazid susceptible disease and 190 days for isoniazid resistant disease.

One hundred and twenty-three $(6 \%)$ patients had isoniazid resistance on initial drug susceptibility testing. Overall, $43(35 \%)$ of 123 patients with initial isoniazid resistance died during treatment, compared with 498 $(28 \%)$ of 1773 patients without isoniazid resistance (odds ratio 1.38, 95\% confidence interval 0.94 to 2.02 ).

Among all patients, 989 (52\%) had known HIV status (404 HIV positive, $585 \mathrm{HIV}$ negative) and 907 (48\%) had unknown HIV status. HIV was not associated with initial isoniazid resistance $(\mathrm{P}=0.6)$ among patients with known HIV status, although it was strongly associated with death (odds ratio 4.20, 3.16 to 5.59$)$.

When restricted to patients with known HIV status, the unadjusted association between isoniazid resistance and death was not significant among HIV positive or HIV negative patients. Specifically, among known HIV positive patients, 16 deaths occurred among 29 patients with isoniazid resistant disease and 181 deaths among 375 patients with isoniazid susceptible disease (odds ratio 1.32, 0.63 to 2.78). Among known HIV negative patients, six deaths occurred among 37 patients with isoniazid resistant disease and 102 deaths among 548 patients with isoniazid

Cases of clinical tuberculous meningitis reported in United States, 1993-2005 ( $n=3114)$

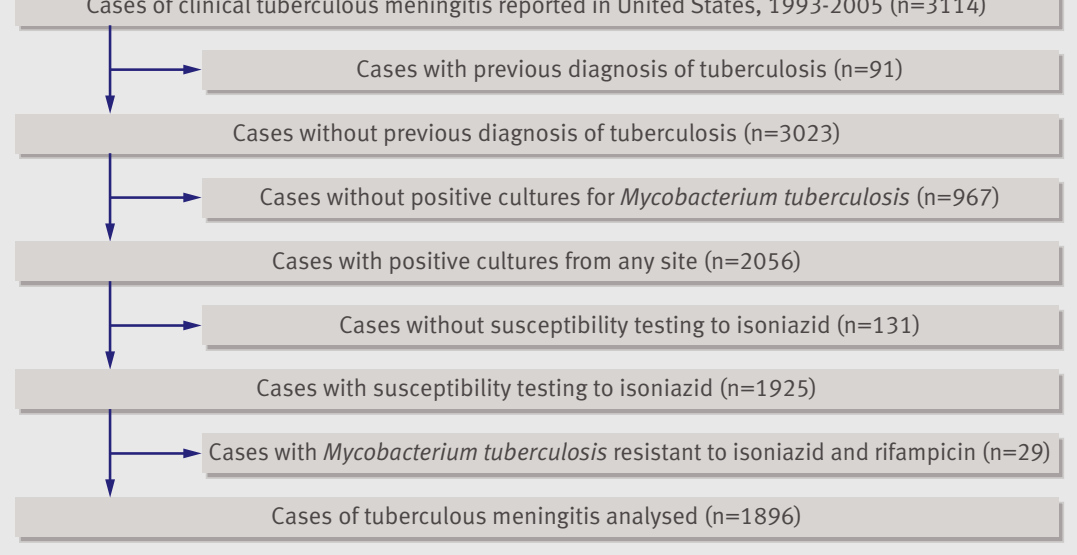

Selection of cases for primary analysis susceptible disease (odds ratio $0.85,0.35$ to 2.03). As both stratum specific associations (odds ratios) included the null value of 1 , known HIV infection was not a significant effect modifier of the relation between initial isoniazid resistance and subsequent death.

In unadjusted analysis stratified by the presence or absence of a positive cerebrospinal fluid culture, the increased risk of death in patients with initial isoniazid resistance was limited to patients with a positive cerebrospinal fluid culture. Among 1614 patients with a positive cerebrospinal fluid culture, 43 (39\%) of 109 with initial isoniazid resistance died during treatment, compared to $433(29 \%)$ of 1505 patients without initial isoniazid resistance (odds ratio 1.61, 1.08 to 2.40). Among the 282 patients with a positive culture from a non-cerebrospinal fluid site, none of the 14 patients with initial isoniazid resistance died (odds ratio 0,0 to 0.87$)$.

\section{Adjusted analyses}

Table 1 shows the characteristics screened for inclusion in the multivariate logistic regression model, the number of non-missing observations for each characteristic, the proportion of patients with each characteristic according to treatment outcome, and the unadjusted odds ratios for the outcome of death during treatment. In the adjusted analysis, advancing age and race were the only significant confounders of the association between initial isoniazid resistance and death. We found no interaction between HIV status and initial isoniazid resistance $(\mathrm{P}=0.7)$. In contrast, a significant interaction existed between isoniazid resistance and positive cerebrospinal fluid cultures $(\mathrm{P}<0.001)$. Among the 1614 patients with positive cerebrospinal fluid cultures, the odds ratio for initial isoniazid resistance and death was 2.07 (1.30 to 3.29). Table 2 shows the individual odds ratios for the other variables among patients with positive cerebrospinal fluid cultures.

\section{Secondary analyses}

As the biological rationale of this investigation rested on the pharmacological properties of the first line agents, we did a secondary analysis of 1050 adults (older than 14 years) with positive cerebrospinal fluid cultures treated initially with isoniazid, rifampicin (or rifabutin), ethambutol, and pyrazinamide (odds ratio for initial isoniazid resistance and subsequent death $2.14,1.25$ to 3.64 ).

Multiple imputation assumes that whether an observation is missing does not depend on unobserved variables. The reporting of HIV status for residents of California occurs only for patients listed in the AIDS registry, and not for patients who tested negative for HIV, which violates this assumption. To test the sensitivity of our results to this mechanism, we repeated the analysis after imputing HIV status for all California residents, without matching to the AIDS registry (odds ratio for initial isoniazid resistance and subsequent death among patients with positive cerebrospinal fluid cultures $2.12,1.34$ to 3.36 ). 
Table 1| Unadjusted analysis of characteristics associated with death $(n=1896)$. Values are numbers (percentages) unless stated otherwise

\begin{tabular}{|c|c|c|c|}
\hline Characteristics & $\begin{array}{l}\text { Death } \\
(n=541)\end{array}$ & $\begin{array}{l}\text { Other outcomes } \\
\quad(n=1355)\end{array}$ & $\begin{array}{l}\text { Unadjusted odds ratio for death } \\
\qquad(95 \% \mathrm{Cl})\end{array}$ \\
\hline \multicolumn{4}{|l|}{ Isoniazid resistance on initial susceptibility testing: } \\
\hline Overall & $43(8)$ & $80(6)$ & $1.38(0.94$ to 2.02$)$ \\
\hline Positive cerebrospinal fluid culture & 43/476 (9) & $66 / 1138(6)$ & 1.61 (1.08 to 2.40$)$ \\
\hline Culture from non-cerebrospinal fluid site & $0 / 65(0)$ & $14 / 217(6)$ & 0 (0 to 0.87$)$ \\
\hline \multicolumn{4}{|l|}{ Age categories (years): } \\
\hline$\leq 1$ & $4(1)$ & $85(6)$ & 0.17 (0.04 to 0.49$)$ \\
\hline$>1$ to $\leq 4$ & $8(1)$ & $114(8)$ & $0.26(0.10$ to 0.56$)$ \\
\hline$>4$ to $\leq 14$ & $6(1)$ & $52(4)$ & $0.43(0.14$ to 1.05$)$ \\
\hline$>14$ to $\leq 24$ & $23(4)$ & $100(7)$ & 0.85 (0.48 to 1.47$)$ \\
\hline$>24$ to $\leq 34$ & $67(12)$ & $247(18)$ & Reference \\
\hline$>34$ to $\leq 44$ & $124(23)$ & $263(19)$ & 1.74 (1.22 to 2.49$)$ \\
\hline$>44$ to $\leq 54$ & $96(18)$ & $188(14)$ & 1.88 (1.29 to 2.76$)$ \\
\hline$>54$ to $\leq 64$ & $61(11)$ & $123(9)$ & $1.83(1.19$ to 2.81$)$ \\
\hline$>64$ to $\leq 74$ & $82(15)$ & $112(8)$ & 2.70 (1.79 to 4.07$)$ \\
\hline$>74$ & $70(13)$ & $71(5)$ & $3.63(2.32$ to 5.69$)$ \\
\hline Race: & $(n=537)$ & $(n=1342)$ & \\
\hline White, non-Hispanic & $85(16)$ & $188(14)$ & Reference \\
\hline Black, non-Hispanic & $231(43)$ & $458(34)$ & $1.12(0.82$ to 1.53$)$ \\
\hline Hispanic & $148(28)$ & $432(32)$ & $0.76(0.55$ to 1.06$)$ \\
\hline Asian/native Hawaiian, non-Hispanic & $62(12)$ & $256(19)$ & 0.54 (0.36 to 0.79$)$ \\
\hline American Indian, non-Hispanic & $11(2)$ & $8(1)$ & 3.04 (1.07 to 9.01$)$ \\
\hline Male sex & $351(65)$ & $799(59)$ & 1.29 (1.05 to 1.58$)$ \\
\hline HIV positive & $197 / 305(65)$ & $207 / 684(30)$ & $4.20(3.16$ to 5.59$)$ \\
\hline \multicolumn{4}{|l|}{ Occupation: } \\
\hline Unemployed & $318 / 421(76)$ & $747 / 1186(63)$ & Reference \\
\hline Healthcare worker & $9 / 421(2)$ & $43 / 1186(4)$ & $0.49(0.21$ to 1.04$)$ \\
\hline Other & $94 / 421(22)$ & $396 / 1186(33)$ & $0.56(0.43$ to 0.73$)$ \\
\hline Injecting drug use within previous year & 44/419 (11) & 49/1192 (4) & $2.74(1.80,4.17)$ \\
\hline Drug use (non-injecting) within previous year & $47 / 410(11)$ & $80 / 1183(7)$ & $1.79(1.22,2.60)$ \\
\hline Alcohol use within previous year & $70 / 406(17)$ & $111 / 1180(9)$ & $2.01(1.45,2.77)$ \\
\hline Homeless within previous year & $29 / 486(6)$ & $57 / 1278(4)$ & $1.36(0.86$ to 2.15$)$ \\
\hline Pulmonary disease & $226(42)$ & $511(38)$ & $1.19(0.97$ to 1.45$)$ \\
\hline Positive acid fast bacilli smear of sputum & $89 / 260(34)$ & $154 / 570(27)$ & 1.41 (1.03 to 1.93$)$ \\
\hline Positive sputum culture & $165 / 246(67)$ & $303 / 531(57)$ & $1.53(1.12$ to 2.10$)$ \\
\hline Abnormal chest radiograph & $321 / 507(63)$ & $720 / 1290(56)$ & $1.37(1.11$ to 1.69$)$ \\
\hline Resident of correctional facility at diagnosis & $20 / 537(4)$ & $30 / 1349(2)$ & 1.70 (0.96 to 3.00$)$ \\
\hline Resident of long term care facility at diagnosis & $26 / 513(5)$ & $55 / 1319(4)$ & $1.23(0.76$ to 1.97$)$ \\
\hline Foreign birth & $185 / 532(35)$ & $591 / 1347(44)$ & $0.68(0.55$ to 0.84$)$ \\
\hline Positive tuberculin skin test & $120 / 275(44)$ & $543 / 880(62)$ & $0.48(0.37$ to 0.63$)$ \\
\hline Positive acid fast bacilli smear from non-sputum specimen & $167 / 470(36)$ & $367 / 1163(32)$ & $1.20(0.95$ to 1.50$)$ \\
\hline Positive cerebrospinal fluid culture & $476(88)$ & $1138(84)$ & 1.40 (1.04 to 1.88$)$ \\
\hline
\end{tabular}

Intravenous drug use, non-intravenous drug use, and alcohol use were all associated with subsequent death in unadjusted analysis, yet these characteristics were not associated with isoniazid resistant disease among patients with known status. In a sensitivity analysis, we found that the potential for unmeasured confounding due to missing observations for these three characteristics was minimal.

Finally, we investigated the extent to which loss to follow-up could have influenced the observed association. Firstly, we excluded 178 patients with an outcome other than completion or death and repeated the steps of the primary analysis (odds ratio $2.22,1.37$ to 3.60 ) Secondly, we introduced non-differential misclassification of outcome among patients lost to follow-up, randomly assigning $50 \%$ of those patients to the outcome of death, regardless of exposure status (odds ratio 1.90, 1.24 to 2.92 ). Thirdly, we introduced differential misclassification of outcome, by randomly re-assigning $25 \%$ of patients lost to follow-up to death in the isoniazid susceptible category but none in the isoniazid resistant category (odds ratio $1.74,1.10$ to 2.76 ). None of these modifications changed the conclusion that initial isoniazid resistance was associated with subsequent 
death among cases of tuberculous meningitis with positive cerebrospinal fluid cultures.

\section{DISCUSSION}

In this national cohort of people with tuberculous meningitis, isoniazid resistance on initial susceptibility testing was associated with subsequent death among those with a positive cerebrospinal fluid culture for $M$ tuberculosis. This association was independent of age and HIV status and remained robust in a secondary analysis limited to adults treated initially with isoniazid, rifampicin (or rifabutin), ethambutol, and pyrazinamide and in sensitivity analyses that considered loss to follow-up.

\section{Comparison with previous studies}

Outcomes of treatment for tuberculous meningitis are poor when resistance to both isoniazid and rifampicin is present. ${ }^{21}$ Isoniazid is unique among the first line anti-tuberculous agents for its dual properties of high penetration of cerebrospinal fluid and bactericidal activity against $M$ tuberculosis. Concentrations of rifampicin in cerebrospinal fluid during treatment for tuberculous meningitis are typically within the range of the minimum inhibitory concentration for $M$ tuberculosis, which may be inadequate for rapid sterilisation of cerebrospinal fluid. ${ }^{4}$ With initial isoniazid resistance, the four drug regimen is left without a bactericidal agent with high levels of cerebrospinal fluid penetration. That the detrimental effect of isoniazid resistant/ rifampicin susceptible disease is intermediate between drug susceptible and multi-drug resistant disease

Table 2 | Multivariate logistic regression model for death among 1614 patients with positive cerebrospinal fluid cultures

\begin{tabular}{|c|c|c|}
\hline Characteristic & Odds ratio $(95 \% \mathrm{Cl})$ & $P$ value \\
\hline Isoniazid resistance & 2.07 (1.30 to 3.29) & 0.002 \\
\hline Male sex & $1.20(0.88$ to 1.63$)$ & 0.243 \\
\hline Age categories (years): & & Overall $<0.001$ \\
\hline$\leq 1$ & $0.076(0.008$ to 0.69$)$ & 0.022 \\
\hline$>1$ to $\leq 4$ & $0.23(0.067$ to 0.82$)$ & 0.023 \\
\hline$>4$ to $\leq 14$ & $0.38(0.102$ to 1.41$)$ & 0.144 \\
\hline$>14$ to $\leq 24$ & $1.22(0.64$ to 2.34$)$ & 0.540 \\
\hline$>24$ to $\leq 34$ & Reference & \\
\hline$>34$ to $\leq 44$ & 1.30 (0.87 to 1.92$)$ & 0.197 \\
\hline$>44$ to $\leq 54$ & 1.97 (1.23 to 3.15$)$ & 0.005 \\
\hline$>54$ to $\leq 64$ & 1.83 (1.09 to 3.09) & 0.023 \\
\hline$>64$ to $\leq 74$ & $4.36(2.48$ to 7.67$)$ & $<0.001$ \\
\hline$>74$ & $6.90(3.85$ to 12.38$)$ & $<0.001$ \\
\hline Race categories: & & Overall $<0.001$ \\
\hline White, non-Hispanic & Reference & \\
\hline Black, non-Hispanic & 1.44 (1.01 to 2.06$)$ & 0.046 \\
\hline Hispanic & 1.21 (0.74 to 1.99$)$ & 0.431 \\
\hline Asian*, non-Hispanic & 0.64 (0.30 to 1.35$)$ & 0.220 \\
\hline American Indian, non-Hispanic & 9.07 (2.65 to 31.02$)$ & $<0.001$ \\
\hline Other†, non-Hispanic & 0.85 (0.20 to 3.52) & 0.819 \\
\hline HIV positive & 3.57 (1.87 to 6.82$)$ & 0.002 \\
\hline
\end{tabular}

*Includes Pacific Islander and native Hawaiian categories.

†includes multiple race and unknown categories. should not be surprising. Our findings also build on an earlier study showing delayed cerebrospinal fluid sterilisation in cases of tuberculous meningitis that are resistant to either isoniazid or streptomycin. ${ }^{22}$

Although reviews of trials from the British Medical Research Council suggested that four drug treatment is an adequate empirical regimen in the setting of isoniazid resistance, ${ }^{1323}$ a recent meta-analysis of a larger number of clinical trials has highlighted the uncertainty in this area. ${ }^{24} \mathrm{We}$ found that isoniazid resistance is a threat to the successful treatment of tuberculosis when meningeal involvement is present. The narrow time window between starting treatment and death, relative to the time needed for culture and susceptibility results, makes the choice of empirical treatment for tuberculous meningitis a critical decision. The use of rapid, molecular assays for drug susceptibility testing would provide earlier identification of drug resistant cases of tuberculous meningitis and should be evaluated further. ${ }^{25}$

\section{Strengths and limitations}

This study had several limitations. Although the exact cause of death is not reported, we believed it to be unlikely that a significant proportion of patients who stopped treatment owing to death would have died for reasons unrelated to the underlying disease process, given the severity of disease characteristic of tuberculous meningitis. We could not measure clinical outcomes other than death during treatment. Survivors of tuberculous meningitis are often left with considerable neurological disability, and we could not assess the effect of initial isoniazid resistance on relevant non-fatal outcomes. ${ }^{26} \mathrm{We}$ also were unable to measure the use of adjunctive corticosteroids, which was associated with decreased mortality in a randomised controlled clinical trial. ${ }^{27}$

We handled missing data by the method of multiple imputation. This approach permits complete data methods for analysis, reduces bias due to missing data, and incorporates imputation error into standard errors. ${ }^{28-30}$ The variable with the greatest degree of missing data was HIV status (unknown in $48 \%$ of cases). Among patients with known HIV status, HIV infection was not associated with initial isoniazid resistance $(\mathrm{P}=0.6)$. Furthermore, HIV status was not a confounder of the association between initial isoniazid resistance and subsequent death. An absence of association between HIV infection and isoniazid resistance has also been noted in studies of all cases of tuberculosis in the surveillance datasets of the United States and the United Kingdom. ${ }^{3132}$ Therefore, unmeasured confounding by HIV status would be unlikely to be strong enough to explain the association between initial isoniazid resistance and subsequent death.

In considering the effect of loss to follow-up, we found that the association of initial isoniazid resistance and subsequent death remained significant even with $25 \%$ differential misclassification of loss to follow-up. This degree of differential misclassification is unlikely given the difference in median duration of treatment 


\section{WHAT IS ALREADY KNOWN ON THIS TOPIC}

The mortality of tuberculous meningitis is higher than that of any other form of tuberculosis

Starting effective anti-tuberculous treatment early is the key to a successful outcome

Isoniazid has pharmacological properties that may promote its effectiveness in the treatment of tuberculous meningitis

\section{WHAT THIS STUDY ADDS}

Initial isoniazid resistance was associated with death during anti-tuberculous treatment in patients with tuberculous meningitis

In areas with a high prevalence of isoniazid resistance among newly diagnosed cases of tuberculosis, treatment strategies other than the standard four drug regimen should be evaluated
Ethical approval: The Institutional Review Board of the University of Pennsylvania approved the study protocol.

Data sharing: No additional data available.

1 Silber E, Sonnenberg P, Ho KC, Koornhof HJ, Eintracht S, Morris L, et al. Meningitis in a community with a high prevalence of tuberculosis and HIV infection. J Neurol Sci 1999;162:20-6.

2 Bergemann A, Karstaedt AS. The spectrum of meningitis in a population with high prevalence of HIV disease. OJM 1996;89:499-504.

3 Thwaites GE, Tran TH. Tuberculous meningitis: many questions, too few answers. Lancet Neurol 2005;4:160-70.

4 Ellard GA, Humphries MJ, Allen BW. Cerebrospinal fluid drug concentrations and the treatment of tuberculous meningitis. Am Rev Respir Dis 1993:148:650-5.

5 Shin SG, Roh JK, Lee NS, Shin JG, Jang IJ, Park CW, et al. Kinetics of isoniazid transfer into cerebrospinal fluid in patients with tuberculous meningitis. J Korean Med Sci 1990;5:39-45.

6 Girgis NI, Yassin MW, Laughlin LW, Edman DC, Farid Z, Watten RH. Rifampicin in the treatment of tuberculous meningitis. J Trop Med Hyg 1978;81:246-7.

between patients who were lost to follow-up and patients who died.

\section{Conclusions and policy implications}

On a global scale, the mortality from tuberculous meningitis attributable to isoniazid resistance will be greater when isoniazid resistance among newly diagnosed cases is more common. ${ }^{33}$ In addition, the relation between initial isoniazid resistance and death may be different in settings without access to second line anti-tuberculous drugs with demonstrated activity in the central nervous system (such as cycloserine or the fluoroquinolones), ${ }^{34}$ or in settings without readily available neurosurgical services. Recommendations for the treatment of tuberculosis suggest the use of an empirically expanded regimen for seriously ill patients when drug resistance is suspected. ${ }^{14}$ Our findings lend strength to this recommendation by showing an increased risk of death in patients with tuberculous meningitis and initial isoniazid resistance. Identification of risk factors for isoniazid resistance among patients with tuberculous meningitis could help clinicians with the choice of empirical treatment. Randomised controlled trials are needed to evaluate the optimal empirical regimen for patients with tuberculous meningitis who are at high risk for both initial isoniazid resistance and poor clinical outcomes.

We thank the local health departments who collected data for this analysis and Valerie Robison, Sandy Althomsons, and Carla Jeffries for their contribution to the analysis. The findings and conclusions in this paper are those of the authors and do not necessarily represent the views of the Centers for Disease Control and Prevention.

Contributors: CV and GPB conceived and designed the study. RRM assisted with literature review. EPW provided guidance on statistical methods. CV did all data analyses, with assistance from CAW. CV wrote the first draft of the manuscript. All authors contributed to the interpretation of the results and revising of the manuscript. CV is the guarantor.

Funding: None.

Competing interests: All authors have completed the Unified Competing Interest form at http://www.icmje.org/coi_disclosure.pdf (available on request from the corresponding author) and declare that (1) no authors have relationships that might have an interest in the submitted work in the previous 3 years; (2) their spouses, partners, or children have no financial relationships that may be relevant to the submitted work; and (3) no authors have non-financial interests that may be relevant to the submitted work.
7 Girgis NI, Yassin MW, Sippel JE, Sorensen K, Hassan A, Miner WF, et al. The value of ethambutol in the treatment of tuberculous meningitis. J Trop Med Hyg 1976;79:14-7.

8 Nau R, Prange HW, Menck S, Kolenda H, Visser K, Seydel JK. Penetration of rifampicin into the cerebrospinal fluid of adults with uninflamed meninges. J Antimicrob Chemother 1992;29:719-24.

9 Ellard GA, Humphries MJ, Gabriel M, Teoh R. Penetration of pyrazinamide into the cerebrospinal fluid in tuberculous meningitis. BMJ 1987;294:284-5.

10 Phuapradit P, Supmonchai K, Kaojarern S, Mokkhavesa C. The blood/cerebrospinal fluid partitioning of pyrazinamide: a study during the course of treatment of tuberculous meningitis. J Neurol Neurosurg Psychiatry 1990;53:81-2.

11 Jindani A, Dore Cl, Mitchison DA. Bactericidal and sterilizing activities of antituberculosis drugs during the first 14 days. Am J Respir Crit Care Med 2003;167:1348-54

12 Donald PR, Sirgel FA, Venter A, Parkin DP, Seifart HI, van de Wal BW, et al. Early bactericidal activity of antituberculosis agents. Expert Rev Anti Infect Ther 2003;1:141-55.

13 Mitchison DA, Nunn AJ. Influence of initial drug resistance on the response to short-course chemotherapy of pulmonary tuberculosis. Am Rev Respir Dis 1986;133:423-30.

14 Treatment of tuberculosis. MMWR Recomm Rep 2003;52:1-77.

15 Rock RB, Olin M, Baker CA, Molitor TW, Peterson PK. Central nervous system tuberculosis: pathogenesis and clinical aspects. Clin Microbiol Rev 2008;21:243-61.

16 Nau R, Sorgel F, Prange HW. Pharmacokinetic optimisation of the treatment of bacterial central nervous system infections. Clin Pharmacokinet 1998;35:223-46.

17 Thwaites GE, Lan NT, Dung NH, Quy HT, Oanh DT, Thoa NT, et al. Effect of antituberculosis drug resistance on response to treatment and outcome in adults with tuberculous meningitis. J Infect Dis 2005;192:79-88.

18 Trends in tuberculosis-United States, 2008. MMWR Morb Mortal Wkly Rep 2009;58:249-53.

19 Curtis AB, McCray E, McKenna M, Onorato IM. Completeness and timeliness of tuberculosis case reporting: a multistate study. $\mathrm{Am}$ Prev Med 2001;20:108-12.

20 Little R, Rubin D. Statistical analysis with missing data. Wiley; 1987

21 Patel VB, Padayatchi N, Bhigjee Al, Allen J, Bhagwan B, Moodley AA, et al. Multidrug-resistant tuberculous meningitis in KwaZulu-Natal, South Africa. Clin Infect Dis 2004;38:851-6.

22 Thwaites GE, Caws M, Chau T, Dung NT, Campbell JI, Phu NH, et al. Comparison of conventional bacteriology with nucleic acid amplification (amplified mycobacterium direct test) for diagnosis of tuberculous meningitis before and after inception of antituberculous chemotherapy. J Clin Microbiol 2004;42:996-1002.

23 Hong Kong Chest Service/British Medical Research Council. Five-year follow-up of a controlled trial of five 6-month regimens of chemotherapy for pulmonary tuberculosis. Am Rev Respir Dis 1987;136:1339-42.

24 Lew W, Pai M, Oxlade O, Martin D, Menzies D. Initial drug resistance and tuberculosis treatment outcomes: systematic review and metaanalysis. Ann Intern Med 2008;149:123-34.

25 Palomino JC. Molecular detection, identification and drug resistance detection in Mycobacterium tuberculosis. FEMS Immunol Med Microbiol 2009;56:103-11.

26 Kalita J, Misra UK, Ranjan P. Predictors of long-term neurological sequelae of tuberculous meningitis: a multivariate analysis. Eur J Neurol 2007;14:33-7.

27 Thwaites GE, Nguyen DB, Nguyen HD, Hoang TQ, Do TT, Nguyen TC, et al. Dexamethasone for the treatment of tuberculous meningitis in adolescents and adults. N Engl J Med 2004;351:1741-51. 
28 Arnold AM, Kronmal RA. Multiple imputation of baseline data in the cardiovascular health study. Am J Epidemiol 2003;157:74-84.

29 Donders AR, van der Heijden GJ, Stijnen T, Moons KG. Review: a gentle introduction to imputation of missing values. J Clin Epidemiol 2006;59:1087-91.

30 Van der Heijden GJ, Donders AR, Stijnen T, Moons KG. Imputation of missing values is superior to complete case analysis and the missing-indicator method in multivariable diagnostic research: a clinical example. J Clin Epidemiol 2006;59:1102-9.

31 Hoopes AJ, Kammerer JS, Harrington TA, ljaz K, Armstrong LR. Isoniazid-monoresistant tuberculosis in the United States, 1993 to 2003. Arch Intern Med 2008;168:1984-92.
32 French CE, Glynn JR, Kruijshaar ME, Ditah IC, Delpech V, Abubakar I. The association between HIV and antituberculosis drug resistance. Eur Respir J 2008;32:718-25.

33 Aziz MA, Wright A, Laszlo A, De Muynck A, Portaels F, Van Deun A, et al. Epidemiology of antituberculosis drug resistance (the Global Project on Anti-tuberculosis Drug Resistance Surveillance): an updated analysis. Lancet 2006;368:2142-54.

34 Thwaites G, Fisher M, Hemingway C, Scott G, Solomon T, Innes J. British Infection Society guidelines for the diagnosis and treatment of tuberculosis of the central nervous system in adults and children. J Infect 2009;59:167-87.

Accepted: 12 July 2010 